\title{
翼 \\ Ocorrência de Giardia spp. em praças públicas de municípios do sul do estado do Rio Grande do Sul, Brasil
}

\author{
[Occurrence of Giardia spp. in public squares of municipalities in the Southern state of Rio Grande \\ do Sul, Brazil]
}

\section{"Artigo Científico/Scientific Article"}

\author{
Alexsander Ferraz ${ }^{1 *}$, Tainá Ança Evaristo ${ }^{1}$, Bruna dos Santos Pires ${ }^{2}$, Diego Moscarelli Pinto ${ }^{1}$, \\ Leandro Quintana Nizoli ${ }^{1}$
}

\begin{abstract}
${ }^{1}$ Departamento de Veterinária Preventiva, Universidade Federal de Pelotas (UFPel), Pelotas-RS, Brasil ${ }^{2}$ Departamento de Clínica Veterinária, Universidade Federal de Pelotas (UFPel), Pelotas-RS, Brasil

*Autor para correspondência/Corresponding author: E-mail: xanderferraz@yahoo.com.br
\end{abstract}

\begin{abstract}
Resumo
O objetivo deste estudo foi avaliar a presença de cistos de Giardia spp. em fezes de cães coletadas em praças de seis municípios do sul do estado do Rio Grande do Sul, Brasil. Entre os meses de setembro de 2017 a março de 2018 foram coletadas 20 amostras mensais em cada município estudado, totalizando 120 amostras mensais e 840 no total. As fezes foram processadas pelo método de Faust (1938). Das amostras analisadas, $153 / 840(18,2 \%)$ continham cistos deste parasito. O município que apresentou maior percentual de amostras positivas foi Cerrito, com um total de 22,9\% (32/140). Os municípios de Pelotas, São Lourenço, Rio Grande, Cassino e Pedro Osório apresentaram 16,4\%, 16,4\%, 17,9\%, 18,6\% e 17,1\%, respectivamente. Pelo fato de serem locais públicos e Giardia apresentar potencial zoonótico, é necessário adotar medidas que visem à diminuição da contaminação dos animais e consequentemente do ambiente, diminuindo a exposição a este protozoário.
\end{abstract}

Palavras-chave: protozoário; zoonose; cães; fezes.

\begin{abstract}
The objective of this study was to evaluate the presence of Giardia spp. cysts in dog feces collected in squares from six municipalities in the Southern state of Rio Grande do Sul, Brazil. Between September 2017 and March 2018, 20 samples were collected monthly in each municipality, totaling 120 monthly samples and 840 in total. The feces were processed by the method described by Faust (1938). Of the samples analyzed, $153 / 840(18.2 \%)$ contained cysts of this parasite. The municipality with the highest percentage of positive samples was Cerrito, with a total of 22.9\% (32/140). The municipalities of Pelotas, São Lourenço, Rio Grande, Cassino, and Pedro Osório, presented 16.4\%, 16.4\%, 17.9\%, 18.6\%, and 17.1\%, respectively. Because they are public places and it is a zoonosis, it is necessary to adopt measures that aim at reducing the contamination of the animals and consequently of the environment, reducing exposure to this protozoan.
\end{abstract}

Keywords: protozoan; zoonosis; dogs; feces.

\section{Introdução}

O cão é considerado cada vez mais como um membro da família. Se por um lado associa-se ao bem-estar psicológico e fisiológico dos seres humanos, pode também representar riscos à saúde pública, pela disseminação de doenças potencialmente zoonóticas (Capuano e Rocha,
2006). Dentre estas, inserem-se as protozooses gastrintestinais, como as transmitidas pelo protozoário Giardia spp. (Overgaauw et al., 2009). A prevalência tende a ser mais elevada em ambientes onde há maior concentração de animais, como em praças e praias (locais públicos). Esta prevalência varia em função das 
populações estudadas, das técnicas utilizadas para diagnóstico coprológico, da experiência do analista e da excreção intermitente dos cistos nas fezes do hospedeiro (Palmer et al., 2008; Epe et al., 2010).

O cisto de Giardia spp. é o estágio que possui resistência ambiental e a fase de transmissão do parasito, podendo imediatamente infectar o hospedeiro susceptível e, geralmente, está presente nas fezes de cães assintomáticos (Carlin et al., 2006). O trofozoíto é a forma ativa do protozoário, encontrada no intestino dos hospedeiros e, está associado à apresentação clínica da doença (Erlandsen et al., 1996). Ocasionalmente, pode ser encontrado nas fezes, principalmente se estas forem diarreicas, pois devido ao aumento do peristaltismo intestinal, os trofozoítos são expelidos antes mesmo de tornarse cisto, não sobrevivendo muito tempo fora do hospedeiro e, por isso, não provoca infecção (Carlin et al., 2006).

O cão contamina-se por coprofagia, ingestão acidental do parasita durante a sua higiene ou por ingestão de água e alimentos contaminados com cistos (Tangtrongsup e Scorza, 2010). As infecções por G. duodenalis no homem estão associadas aos assemblages $\mathrm{A}$ ou $\mathrm{B}$, sendo que o assemblage A é frequente em cães, ocasionalmente pode ser encontrado o assemblage B (Manser et al., 2013; Zanzani et al., 2014).

Em cães, a giardíase é geralmente assintomática ou autolimitante (Thompson et al., 2008). As manifestações clínicas estão associadas a cães imunocomprometidos ou portadores de doenças crônicas, principalmente se estiverem presentes infecções concomitantes (Tangtrongsup e Scorza, 2010), nestes casos pode ocorrer manifestação com gravidade e elevada morbidade, ou evoluir para quadro crônico (Morgan et al., 2000). É mais prevalente em animais jovens, principalmente até 1 ano de idade (26 a 50\%), em canis o protozoário pode ser encontrado, dependendo da higiene do local, em até $100 \%$ dos animais (Mundim et al., 2003). Em contrapartida, a prevalência em felinos varia de 5,9\% até $31,2 \%$ (Almeida et al., 2007; Funada et al., 2007; Coelho et al., 2009; Brinker et al., 2009).

O Centro para a Segurança Alimentar e Saúde Pública (The Center for Food Security \& Public Health, 2012) recomenda tratar os animais sintomáticos para controlar os sinais clínicos, dando suporte ao animal. A eliminação da infecção nos animais assintomáticos, por vezes frustrante, torna-se um objetivo secundário e de discutível necessidade (Fiechter et al., 2012).

O tratamento farmacológico, por si só, não parece ser suficiente para a eliminação do protozoário na sua totalidade. As causas do insucesso terapêutico incluem reinfecção, coinfecção, existência de uma doença subjacente desconhecida ao clínico veterinário ou a persistência do agente após o tratamento, por exemplo, devido à resistência ao fármaco (ESCCAP, 2011).

Diversos pesquisadores, inclusive no Brasil, obtiveram dados de estudos que comprovam que praças e praias apresentam risco potencial de contaminação por parasitoses zoonóticas (Devera et al., 2008).

O presente estudo teve como objetivo relatar a ocorrência de Giardia spp. em fezes de cães coletadas em praças de munícipios do extremo Sul do Rio Grande do Sul, Brasil.

\section{Material e Métodos}

O estudo foi realizado de setembro de 2017 a março de 2018. Mensalmente, foram coletadas 20 amostras viáveis (recentes) de fezes, presentes em praças públicas de seis municípios do extremo sul do estado do Rio Grande do Sul: Pelotas (Latitude: $31^{\circ} 46^{\prime} 19^{\prime \prime} \mathrm{S}$, Longitude: $52^{\circ} 20^{\prime} 33^{\prime \prime}$ W), São Lourenço do Sul (Latitude: $31^{\circ} 21^{\prime} 55^{\prime \prime} \mathrm{S}$, Longitude: $51^{\circ} 58^{\prime} 42^{\prime \prime} \mathrm{W}$ ), Rio Grande (Latitude: $32^{\circ} 02^{\prime} 06^{\prime \prime} \mathrm{S}$, Longitude: $52^{\circ} 05^{\prime} 55^{\prime \prime} \mathrm{W}$, Cassino (Latitude : -32 11' 30" S, Longitude: -52 09' 30" $\mathrm{W}$ ), Pedro Osório (Latitude: $31^{\circ} 51^{\prime} 51^{\prime \prime} \mathrm{S}$, Longitude: $52^{\circ} 49^{\prime} 24^{\prime \prime} \mathrm{W}$ ) e Cerrito (Latitude: $31^{\circ}$ 51' 23" S, Longitude: $52^{\circ} 48^{\prime} 46^{\prime \prime} \mathrm{W}$ ), totalizando 120 amostras mensais e 840 durante todo o estudo.

As amostras fecais foram coletadas em embalagens plásticas, identificadas de acordo com a praça e mantidas em recipientes isotérmicos com gelo retornável, sendo encaminhadas para o Laboratório de Doenças Parasitárias (LADOPAR), localizado na Faculdade de Medicina Veterinária (FaVet), da Universidade Federal de Pelotas (UFPel).

As amostras foram submetidas à técnica de Faust (1938), que emprega o uso de sucessivas centrifugações de uma amostra macerada com água destilada e por final uma última centrifugação com uso de uma solução hipersaturada a base de sulfato de zinco a $33 \%$. Após a elaboração da técnica, as lâminas foram confeccionadas e coradas com lugol. A análise foi 
realizada em microscopia óptica com aumento de 400x.

\section{Resultados}

Dentre as amostras analisadas, 153/840 $(18,2 \%)$ foram positivas para Giardia spp. As amostras negativas totalizaram $687 / 840(81,8 \%)$. O município que apresentou maior ocorrência de contaminação foi Cerrito, com um total de $22,9 \%$ $(32 / 120)$ e o mês que apresentou maior percentual de contaminação foi janeiro, com 20,0\% (24/120) (Tabela 1).

Tabela 1. Frequência de Giardia spp. em amostras fecais de cães coletadas em praças de municípios do extremo sul do Rio Grande do Sul, Brasil.

\begin{tabular}{ccccccccc}
\hline Cidade & Set.17 & Out.17 & Nov.17 & Dez.17 & Jan.18 & Fev.18 & Mar.18 & $\%$ Total \\
\hline Pelotas & $10 \%$ & $15 \%$ & $20 \%$ & $15 \%$ & $20 \%$ & $20 \%$ & $15 \%$ & $16,4(23 / 140)$ \\
São Lourenço & $10 \%$ & $20 \%$ & $15 \%$ & $10 \%$ & $25 \%$ & $15 \%$ & $20 \%$ & $16,4(23 / 140)$ \\
Rio Grande & $15 \%$ & $15 \%$ & $20 \%$ & $15 \%$ & $15 \%$ & $20 \%$ & $25 \%$ & $17,9(25 / 140)$ \\
Cassino & $5 \%$ & $15 \%$ & $20 \%$ & $25 \%$ & $25 \%$ & $20 \%$ & $20 \%$ & $18,6(26 / 140)$ \\
Pedro Osório & $20 \%$ & $15 \%$ & $20 \%$ & $15 \%$ & $15 \%$ & $20 \%$ & $15 \%$ & $17,1(24 / 140)$ \\
Cerrito & $30 \%$ & $20 \%$ & $20 \%$ & $30 \%$ & $20 \%$ & $20 \%$ & $20 \%$ & $22,9(32 / 140)$ \\
\hline Total & 15,0 & 16,7 & 19,2 & 18,3 & 20,0 & 19,2 & 19,2 & $18,2(153 / 840)$ \\
\hline
\end{tabular}

\section{Discussão}

Neste estudo, constatou-se que $18,2 \%$ das amostras analisadas, continham cistos do protozoário Giardia spp. Alguns autores encontraram valores mais baixos, comparados com o presente trabalho, como Campos Filho et al. (2008), que observaram que $0,8 \%$ das 119 amostras de fezes de cães coletadas em praças públicas do município de Itabuna, Bahia, eram positivas para este protozoário, e por Vital et al. (2012), que constataram que das 40 amostras fecais coletadas nas ruas do Distrito Federal, 10,0\% continham cistos de Giardia spp.

Capuano e Rocha (2006), verificando ocorrência de parasitos zoonóticos em fezes de cães colhidas em áreas públicas do município de Ribeirão Preto, constatou que 10,0\% das amostras apresentavam-se positivas para Giardia spp. Ribeiro et al. (2013), analisando 104 amostras provenientes de praças públicas de Belo Horizonte, MG, observou positividade em 15,4\% destas.

Porém, em alguns estudos, observou-se valores mais altos, como Alves et al. (2016), que analisando fezes de cães coletadas em logradouros do município de Pindamonhangaba-SP, encontraram 51,6\% de amostras positivas. Rosales e Malheiros (2017) analisando a contaminação ambiental no município de Cáceres, no Mato Grosso, observaram um total de $18,3 \%$ de amostras positivas para cistos de Giardia spp., percentual próximo aos $18,2 \%$ observado no presente estudo.

A prevalência de giardíase é maior em populações de cães e gatos confinados, como em abrigos por exemplo, devido ao saneamento precário e à superlotação destes locais (Lindsay e Zajac, 2009).

O crescente número de animais domiciliados, peridomiciliados e errantes, associado ao fácil acesso aos locais de lazer, como praças públicas, aumentam os riscos de contaminação para os humanos, principalmente crianças, pois várias espécies de parasitos potencialmente zoonóticos, como é o caso de Giardia spp., podem ser eliminados através das fezes dos animais, constituindo um problema de saúde pública (Castro et al., 2005).

As patologias gastrintestinais de origem parasitária estão intimamente ligadas às condições sanitárias dos locais onde a população humana circula, representando um importante problema em países com subdesenvolvimento, podendo gerar déficit nutricional em crianças, prejudicando o desenvolvimento infantil (Guergel et al., 2005).

$\mathrm{Na}$ Austrália, foi relatada a presença de cães em área urbana parasitados por Giardia de genótipo A (zoonótico) e de genótipo D (específico em cães). Isso demonstra a possibilidade de transmissão de Giardia spp. entre os cães e humanos (Bugg et al., 1999).

Pelas várias fontes de infecção, alta infectividade e resistência dos cistos, a giardíase é uma enteroparasitose de difícil erradicação (Bica et al., 2011).

\section{Conclusão}

A partir dos resultados observados neste estudo, conclui-se que há uma ocorrência considerável de Giardia spp. nas amostras de fezes coletadas em praças dos municípios do sul do Rio Grande do Sul. Pelo fato de apresentar 
potencial zoonótico, torna-se imprescindível a adoção de medidas sanitárias que visem reduzir a contaminação do ambiente, reduzindo a infestação dos animais e humanos.

\section{Conflito de Interesse}

Os autores declaram não existir conflito de interesse.

\section{Comitê de Ética}

Não houve necessidade de aprovação pelo Comitê de Ética, visto que a metodologia do trabalho se baseou em coletas de amostras fecais ambientais.

\section{Referências}

Almeida, F.M.; Silva, M.M.O.; Labarthe, N. Giardia spp. em amostras fecais de gatos domésticos do Rio de Janeiro, RJ. Acta Scientiae Veterinariae, 35(2): 468-469, 2007.

Alves, A.P.S.M.; Coelho, M.D.G.; Santos, I.A.; Bozo, L.S.O.; Maciel, L.T.R. Contaminação em logradouros do município de Pindamonhangaba-SP, por parasitos potencialmente zoonóticos em fezes caninas. Revista de Ciências da Saúde, 1(1): 45-50, 2016.

Bica, V.C.; Dillenburg, A.F.; Tasca, T. Diagnóstico laboratorial da giardiose humana: comparação entre as técnicas de sedimentação espontânea em água e de centrifugo-flutuação em solução de sulfato de zinco. Revista HCPA, 31(1): 39-45, 2011.

Brinker, J.C.; Teixeira, M.C.; Araujo, F.A.P. Ocorrência de Giardia sp. em cães e gatos no município de Caxias do Sul, RS. Revista da FZVA, 16(1): 113-119, 2009.

Bugg, R.J.; Robertson, I.D.; Elliot, A.D.; Thompson, R.C.A. Gastrointestinal parasites of urban dogs in Perth, Western Australia. Veterinary Journal, 157(3): 295-301, 1999.

Campos Filho, P.C.; Barros, L.M.; Campos, J.O.; Braga, V.B.; Cazorla, I.M.; Albuquerque, G.R.; Carvalho, S.M.S. Parasitas zoonóticos em fezes de cães em praças públicas do município de Itabuna, Bahia, Brasil. Revista Brasileira de Parasitologia Veterinária, 17(4): 206-209, 2008.

Capuano, D.M.; Rocha, D.M. Ocorrência de parasitas com potencial zoonótico em fezes de cães coletadas em áreas públicas do município de Ribeirão Preto, SP, Brasil. Revista
Brasileira de Epidemiologia, 9(1): 81-6, 2006.

Carlin, E.P., Bowman, D.D.; Scarlett, J.M. Prevalence of Giardia in symptomatic dogs and cats in the United States. Compendium on Continuing Education for the Practising Veterinarian, 28(11A): 1-12, 2006.

Castro, J.M.; Santos, S.V.; Monteiro, N.A. Contaminação de canteiros da orla marítima do município de Praia Grande, São Paulo, por ovos de Ancylostoma e Toxocara em fezes de cães. Revista da Sociedade Brasileira de Medicina Tropical, 38(2): 199-201, 2005.

Coelho, W.M.D.; Amarante, A.F.T.; Soutello, R.V.G.; Meireles, M.V.; Bresciani, K.D.S. Occurrence of gastrointestinal parasites in fecal samples of cats in Andradina City, São Paulo. Revista Brasileira de Parasitologia Veterinária, 18(2): 46-49, 2009.

Devera, R.; Blanco, Y.; Hernandéz, H.; Simões, D. Toxocara spp. y otros helmintos en plazas y parques de Ciudad Bolívar, estado Bolívar (Venezuela). Enfermedades Infecciosas $\mathbf{y}$ Microbiologia Clínica, 26(1): 23-26, 2008.

Epe, C., Rehkter, G., Schnieder, T., Lorentzen, L.; Kreienbrock, L. Giardia in symptomatic dogs and cats in Europe: Results of a European study. Veterinary Parasitology, 173(1-2): 3238, 2010.

Erlandsen, S.L., Macechko, P.T., Keulen, H.V.; Jarrol, E.L. Formation of the Giardia cyst wall: Studies on extracellular assembly using immunogold labeling and high resolution field emission SEM. Journal of Eukariotic Microbiology, 43(5): 416- 429, 1996.

ESCCAP. European Scientific Counsel Companion Animal Parasites. Control of intestinal protozoa in dogs and cats. ESCCAP Guidelines $\mathbf{n}^{\mathbf{0}}$ 6. $6^{\text {th }}$ ed. Worcestershire, UK: ESCCAP, 2011.

Faust, E.C.; D'antonio, J.S.; Odom, V.; Miller, M.J.; Peres, C.; Sawitz, W.; Thomen, L.F.; Tobie, J.; Walker, J.H. A critical study of clinical laboratory techniques for the diagnosis of protozoan cyst and helminth egg in feces. American Journal Tropical Medicine, 18(2): 169-83, 1938.

Fiechter, R., Deplazes, P.; Schnyder, M. Control of Giardia infections with ronidazole and intensive hygiene management in a dog kennel. Veterinary Parasitology, 187(1-2): 93-98, 2012. 
Funada, M.R.; Pena, H.F.J.; Soares, R.M.; Amaku, M.; Gennari, S.M. Frequência de parasitos gastrintestinais em cães e gatos atendidos em hospital-escola veterinário da cidade de São Paulo. Arquivo Brasileiro de Medicina Veterinária e Zootecnia, 59(5): 1338-1340, 2007.

Guergel, R.G., Cardoso, G.S.; Silva, A.M.; Santos, L.N.; Oliveira, R.C.V. Creche: ambiente expositor ou protetor nas infecções por parasitas intestinais em Aracajú, SE. Revista Brasileira de Medicina Tropical, 38(3): 267- 269, 2005.

Lindsay, D.S.; Zajac, A.M. The biology and control of Giardia spp. and Tritrichomonas foetus. Veterinary Clinics of North America: Small Animal Practice, 39(6): 993-1007, 2009.

Manser, M., Granlund, M., Edwards, H., Saez, A., Petersen, E., Evengard, B.; Chiodini, P. Detection of Cryptosporidium and Giardia in clinical laboratories in Europe: a comparative study. Clinical Microbiology and Infection, 20(1): 65-71, 2013.

Morgan, U.M.; Xiao, L.; Monis, P.T., Fall, A.; Peter, J.; Fayer, R.; Denholm, K.; Limor, J.; Altaf Lal, R.C.; Thompson, A. Cryptosporidium spp. in domestic dogs: The dog genotype. Applied and Environmental Microbiology, 66(5): 2220-2223, 2000.

Mundim, M.J.S.; Souza, S.Z; Hortêncio, S.M; Cury, M.C. Frequência de Giardia spp. por duas técnicas de diagnóstico de fezes em cães. Arquivo Brasileiro de Medicina Veterinária e Zootecnia, 55(6): 770-773, 2003.

Overgaauw, P.; Zutphen, L.; Hoek, D.; Yaya, F.; Roelfsema, J.; Pinelli, E.; Knapen, F.; Kortbeek, L. Zoonotic parasites in fecal samples and fur from dogs and cats in The Netherlands. Veterinary Parasitology, 163(12): 115-122, 2009.
Palmer, C.S.; Traub, R.J.; Robertson, I.D.; Devlin, G.; Rees, R.; Thompson, R.C.A. Determining the zoonotic significance of Giardia and Cryptosporidium in Australian dogs and cats. Veterinary Parasitology, 154(1-2): 142-147, 2008.

Ribeiro, L.M.; Dracs, R.M.; Mozzer, L.R.; Lima, W.S. Soil contamination in public squares in Belo Horizonte, Minas Gerais, by canine parasites in different developmental stages. Revista do Instituto de Medicina Tropical de São Paulo, 55(4): 229-231, 2013.

Rosales, T.F.L.; Malheiros, A.F. Contaminação Ambiental por enteroparasitas presentes em fezes de cães em uma região do Pantanal. O Mundo da Saúde, 41(3): 368-377, 2017.

Tangtrongsup, S.; Scorza, V. Update on the diagnosis and management of Giardia spp infections in dogs and cats. Topics in Companion Animal Medicine, 25(3): 155162, 2010.

The Center for Food Security \& Public Health. (2012). Giardiasis. Disponível em: <http://www.cfsph.iastate.edu/Factsheets/pdfs/ giardiasis.pdf >. Acesso em: 20 fev. 2018.

Thompson, R.C.A.; Palmer, C.S.; Handley, R.O. The public health and significance of Giardia and Cryptosporidium in domestic animals. The Veterinary Journal, 177(1): 18-25, 2008.

Vital, T.E.; Barbosa, M.R.A.; Mendes, D.S. Ocorrência de parasitos com potencial zoonótico em fezes de cães e gatos do Distrito Federal. Ensaios e Ciências: Ciências Biológicas, Ágrarias e da Saúde, 16(1): 9-23, 2012.

Zanzani, S.A.; Gazzonis, A.L.; Scarpa, P., Berrilli, F.; Manfredi, M.T. Intestinal parasites of owned dogs and cats from metropolitan and micropolitan areas: Prevalence, zoonotic risks, and pet owner awareness in northern Italy. BioMed Research International, 2014:1-10, 2014. 